\title{
Enhance contrast in PCA based beamformers using smoothing kernel
}

\author{
Saeid Aliabadi ${ }^{\mathrm{a}}$, Jinhua $\mathrm{Yu}^{\mathrm{a}, \mathrm{b}}$ and Yuanyuan Wang, ${ }^{\mathrm{a}, \mathrm{b}, *}$ \\ ${ }^{a}$ Department of Electronic Engineering, Fudan University, Shanghai 200433, China \\ ${ }^{b}$ Key laboratory of Medical Imaging Computing and Computer Assisted Intervention of Shanghai, \\ Shanghai 200433, China
}

\begin{abstract}
The contrast and resolution have trade-off in medical ultrasound imaging. Most of adaptive beamformer can enhance the imaging resolution significantly but not improve the contrast at the same time. The principal component analysis (PCA) based beamformers such as the eigenspace-based minimum variance (ESBMV) beamformer provide a good imaging resolution. Neighbors of the focal point include the common noise, interface and signal components. Echo signal of the neighbor points can be used to suppress the noise and extract the signal component of the focal point. Based on this idea, in order to improve the quality of PCA based beamformers both in the imaging contrast and resolution, a novel beamforming method is proposed. This proposed beamformer utilizes a kernel to select neighbor points. The number of eigenvectors is estimated by using any PCA method. Then the number of selected eigenvectors for each focal point is compared with the number of selected eigenvectors of its neighbor points and is changed to a new value. The selected eigenvectors of the covariance matrix is used to construct the signal subspace. The estimated signal subspace is projected onto the minimum variance (MV) weight vector to calculate the desire weight vector. Results of experiments show that the proposed beamformer can improve the imaging contrast significantly while keeping the resolution quality similar to ESBMV beamformer.
\end{abstract}

Keywords: Ultrasound imaging, beamforming, minimum variance, eigenvalue

\section{Introduction}

Beamforming is a signal processing technique to enhance the image quality by combining echo signals from different angles. To improve the imaging resolution, adaptive beamforming methods have been widely used. Most of adaptive beamformers are based on the adaptive minimum variance (MV) method that proposed by Capon in 1969 [1]. The weights are calculated and the output signal-to-noise ratio (SNR) must be maximized while the target signals remain undistorted. The adaptive beamformer, by changing the weights of array elements or aperture using the characteristics of receive signals, has a beam pattern with lower side lobe levels and the same main lobe width compared with conventional beamformers.

Many methods have applied adaptive beamformers to medical ultrasound imaging and demonstrated the improved image contrast and/or resolution. The MV is a well-known beamformer due to its

\footnotetext{
* Address for correspondence: Yuanyuan Wang, Department of Electronic Engineering, Fudan University, Shanghai 200433, China. Tel./Fax: +86 21 65643526; E-mail: yywang@fudan.edu.cn.
}

0959-2989/15/\$35.00 @ 2015 - IOS Press and the authors. 
benefits of increasing resolution and frame rate [2]. Sasso and Cohen Bacire [3] applied a spatial smoothing technique to the covariance matrix to improve the estimation robustness of the covariance matrix. Synnevag, et al. [4] proposed a temporal averaged MV beamformer, in which temporal averaging and spatial averaging have been used. Temporal averaging improves the accuracy of the covariance matrix. They also used diagonal loading to make the covariance matrix more robust. B. Mohammadzade, et al. [5] applied the eigenspace-based MV (ESBMV) beamformer to medical ultrasound imaging to improve the imaging resolution and contrast simultaneously. ESBMV beamformer uses the eigenstructure of the covariance matrix to enhance the image quality. The aperture weights of ESBMV beamformer is founded by projecting the MV beamformer weight vector on to a vector sub space that constructed from the eigenstructure of the covariance matrix. They proposed utilized forward-backward minimum variance beamforming to enhance the imaging contrast and robustness of covariance matrix [6]. S. Mehdizadeh, et al. [7] proposed a new ESBMV and applied it to improve the surface imaging quality. In this method, the cross-spectral metric definition is used to select the number of eigenvectors. A. Rabinovich, et al. [8] combined the multi-line transmission with minimum variance to reduced cross-talk artifacts, without compromising spatial resolution, and even improving it.

The performance of ESBMV beamformer is achieved at the cost of the high computational complexity. Large amount of computation associated with the covariance matrix inversion increase the calculation complexity of ESBMV beamformers. Some methods have been presented to reduce the calculation complexity. X. Zeng, et al. [9] proposed a B-ESBMV beamformer that applied the ESBMV beamformer in the beam domain instead of the array domain. Their method reduces the calculation time while remains the imaging quality. K. Kim, et al. [10] used the principal component analysis (PCA) to reduce the computational complexity. Here the weight vector was calculated approximately. B.M. Asl, et al. [11] using the structured covariance matrix to reduce calculation complexity of minimum variance beamforming methods.

In this paper, we proposed a novel beamforming method by utilizing the common properties of echo signals from neighbors of the focal point. Here a kernel is used to select neighbor points of each focal point. The weight vector of the proposed beamformer is estimated by projecting the MV weight vector onto the signal subspace. Experimental results on simulation and phantom study show that the proposed beamformer can enhance the performance of ESBMV and MV beamformers in terms of the imaging contrast significantly. The outline of this paper is as follows. Background is described in section 2 contains the model of sensor signals, MV and ESBMV beamformers. The proposed method is introduced in section 3. Experimental results are illustrated in section 4. Conclusion and discussion are given in section 5 .

\section{Background}

\subsection{Minimum variance beamformer}

Consider the signal-to-interference pulse-noise ratio (SINR) [12]:

$$
\operatorname{SINR}=\frac{\sigma_{S}^{2}\left|w^{H} a\right|^{2}}{w^{H} R w}
$$

where $\sigma_{s}^{2}$ is the power of the signal, $R$ the $M * M$ covariance matrix, $w$ the weight vector, $a$ the 
steering vector and $(.)^{H}$ the conjugate transpose. The optimum weight vector can be achieved by maximizing the SINR. It can be given by minimizing the output interference-pulse-noise power subject to the receiving signal with no distortion. Maximizing the SINR is equivalent to:

$$
\min w^{H} R w, \text { subject to } w^{H} a=1
$$

Eq. (2) can be solved by utilizing the Lagrange method, and the optimal weight vector can be given by:

$$
w_{0}=\frac{R^{-1} a}{a^{H} R^{-1} a}
$$

\subsection{Eigen space-based minimum variance beamformers}

For a focal point, the sensor signals are pre-steered by applying the MV delays. The received signal at the time $k$ is given by:

$$
x(k)=s(k) a+i(k)+n(k)
$$

where $s(k) a$ is the signal wave form and its steering vector, $i(k), n(k)$ are interface and noise components respectively. By using the spatial smoothing [3] and Eq. (4), the covariance matrix is found by:

$$
R=\frac{1}{N} \sum_{n=1}^{N} x_{n} x_{n}^{H}
$$

where $N=M-L+1$ is the number of overlapping subarrays, $M$ the number of elements, $L$ the dimension of a subarray, and $x_{n}=\left[x_{n+1}(k), \ldots, x_{N+L-1}(k)\right]^{T}$ is the signal vector of $n^{\text {th }}$ subarray. In the diagonal loading factor technique, a constant is added to the diagonal vector of the covariance matrix as $R_{D L}=R+\in \mathrm{I}$, where I is identity matrix, $\mathrm{R}$ denotes covariance matrix and $R_{D L}$ is diagonal loaded covariance matrix. $\in$ is the diagonal loading factor and it can be set as $\mu$ times the power of the received signals [4].

$$
\epsilon=\mu * \operatorname{trace}(R)
$$

where $\operatorname{trace}(R)$ is the Sum of diagonal elements of matrix $R$.

Eigenvalues and eigenvectors of the covariance matrix are formed by:

$$
R_{D L}=E \Lambda E^{H}=\sum_{i=1}^{L} \lambda_{i} e_{i} e_{i}^{H}
$$

where (.) $)^{H}$ denote conjugate transpose, $\Lambda$ is eigenvalue matrix, $\lambda_{1} \geq \lambda_{2} \geq \cdots \geq \lambda_{L}$ are eigenvalues and $e_{i}$ is the relevant eigenvector. The signal subspace is constructed by using eigenvectors corresponding to selected eigenvalues.

$$
E_{S}=\left[e_{1}, e_{2}, \ldots, e_{n s l}\right]
$$


The weigh vector is given by projecting the MV weight vector to the signal subspace:

$$
W_{p c a}=\mathrm{E}_{s} E_{s}^{H} W_{M V}
$$

Eq. (9) can de expressed as projecting the weight vector of MV method $\left(W_{M V}\right)$ into signal subspace of covariance matrix $\mathrm{R}\left(\mathrm{E}_{s}\right)$ to obtain the weight vector of PCA methods $\left(W_{p c a}\right)$.

The weight vector is applied to the received data for each channel and finally all channel's signals are summed up. Some methods were proposed to select the number of eigenvectors $(n s l)$. The selection of the eigenvalue number can be considered by the condition [5]:

$$
\lambda_{n s l}>\partial \lambda_{\max }
$$

where $\lambda_{n s l}$ is the eigenvalue corresponding to eigenvector that is used to construct the signal subspace, $\lambda_{\max }$ is maximum eigenvalue and $\partial$ is a real value multiplier. In [6] the selection of eigenvalue number is given by:

$$
\left(\sum_{k=n s l}^{L} \frac{p_{k}^{2}}{\lambda_{k}}\right)^{-1}<\beta\left(\sum_{k=1}^{L} \frac{p_{k}^{2}}{\lambda_{k}}\right)^{-1}
$$

where $\frac{p_{k}^{2}}{\lambda_{k}}$ is the cross-spectral metric for the time $k, L$ is the subarray length and $\left(\sum_{k=1}^{L} \frac{p_{k}^{2}}{\lambda_{k}}\right)^{-1}$ is the total output signal power.

\section{Proposed method}

In order to improve the imaging contrast, we proposed a method based on the PCA based methods. Since echo signals of near points contain the common interface and noise information, noise and interface signals can be rejected by using the common data of near point's echoes. This is the main idea of this research. In this method, any PCA based method is used to calculate the number of eigenvectors at first. Then, the selected eigenvectors corresponding to neighbor points of each focal point were compared to each other. By using the comparison result, a new eigenvector is used for the focal point. Results show that the proposed method enhances the overall quality of images significantly compared with MV and ESBMV beamformers.

In eigenspace-based methods, the conditions used to select the number of eigenvectors, may cause some signal components lost and keep some interface components. To improve the accuracy of the eigenvector selection, this paper proposes a new method by using the signal subspace dimension of neighbor points to correct the signal subspace dimension of the focal point. For choosing the neighbor points, a kernel is defined. Any PCA based beamformer can be applied to estimate the dimension of the signal subspace of each focal point. After that the number of eigenvectors of kernel points is compared with each other. With this comparison the number of eigenvectors is changed to new corrected value.

A kernel is defined to determine the neighbor points. Figure 1 illustrates the kernel structure. Neighbor points are three points that pre-calculated. The distance between the focal point and two neighbor points are $m * d_{x}, m * \sqrt{d_{x}^{2}+d_{z}^{2}}$ and $m * d_{z}$ respectively. Here $d_{x}$ and $d_{z}$ are the lateral 
and axial resolution distance, respectively, $m \in N$ is a multiplier.

Assume $n s l 1, n s l 2, n s l 3$ and $n s l \mathrm{~F}$ as the number of selected eigenvectors (the dimension of the signal subspace) and $k 1, k 2, k 3$ and $k \mathrm{~F}$ as the time, the corresponding point 1, point 2, point 3 and focal point were estimated by the ESBMV method respectively. The kernel variance is defined as:

$$
\operatorname{var}=\sqrt{\left(y_{\mathrm{pca}}(k \mathrm{~F})-y_{\mathrm{pca}}(k 1)\right)^{2}+\left(y_{\mathrm{pca}}(k \mathrm{~F})-y_{\mathrm{pca}}(k 2)\right)^{2}+\left(y_{\mathrm{pca}}(k \mathrm{~F})-y_{\mathrm{pca}}(k 3)\right)^{2}}
$$

where $y_{\mathrm{pca}}$ is the image value, If the kernel variance is smaller than variance threshold, the minimum value of $n s l 1, n s l 2, n s l 3$ and $n s l \mathrm{~F}$ will be considered as the dimension of the signal subspace for the focal point. In other words, that is:

$$
\text { If } \operatorname{var}<\Delta * \max \left(y_{\mathrm{pca}}\right) \text { then } n s=\min (n s l 1, n s l 2, n s l 3, n s l F)
$$

where $\Delta$ is a multiplier and $\max \left(y_{\mathrm{pca}}\right)$ is the maximum observed image value $\mathrm{In}$ this way we reject the extra eigenvectors corresponding to interface components that may be selected by the ESBMV method.

If the kernel variance is larger than $\Delta$ time image value in focal point, the maximum value of $n s l 1$, $n s l 2, n s l 3$ and $n s l \mathrm{~F}$ will be selected as the dimension of the signal subspace for the focal point. In other words, that is:

$$
\text { If } \operatorname{var}>\Delta * \max \left(y_{\mathrm{pca}}\right) \text { then } n s=\max (n s l 1, n s l 2, n s l 3, n s l F)
$$

In this way we keep the eigenvectors corresponding to signal components that may not be selected by the ESBMV beamformer.

The enhanced signal subspace contains $n s$ number of eigenvectors:

$$
V_{s}=\left[e_{1}, e_{2}, \ldots, e_{n s}\right]
$$

The enhanced weight vector for the proposed method is estimated by projecting the enhanced signal subspace to the MV vector:

$$
W_{p}=\mathrm{V}_{s} * V_{s}^{H} W_{M V}
$$

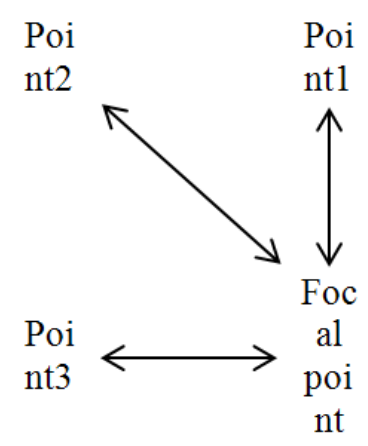

Fig. 1. The structure of the kernel to select neighbor points of each focal point. 


\section{Results}

The MV covariance matrix was calculated and its eigenvalue and eigenvectors were estimated. For each focal point by using the condition of the ESBMV method, the number of eigenvector was calculated. The number of selected eigenvectors was compared with its relevant kernel points. Then new number of eigenvalues was calculated. In proposed method, the value of selected eigenvectors of kernel points (neighbor points of focal point) was calculated before focal point, thus in this way, the calculation time is kept approximately same calculation time of ESBMV beamformer.

\subsection{Simulation study}

In this section some simulation results are given to compare the performance of the proposed beamformer with the MV and ESBMV beamformer. Field II software [13] is used for the simulation with a $19 \mathrm{~mm}$ linear array with 128 elements spaced at one-half wavelength. The central and sampling frequency have been set to $5 \mathrm{MHZ}$ and $100 \mathrm{MHZ}$ respectively.

\subsubsection{Point target simulation}

Point target simulation is used to compare the resolution of proposed method with MV and ESBMV beamformers. To this end 8 points located at depth from $20 \mathrm{~mm}$ to $40 \mathrm{~mm}$. Results are shown in Figure 2. It can be seen that the proposed method improves the imaging resolution of ESBMV method. Imaging resolution is improved in non-target points significantly specially at depths $22 \mathrm{~mm}$ and 42 $\mathrm{mm}$. These points are pointed by arrow.

\subsubsection{Cyst phantom simulation}

To evaluate the contrast enhancement of the beamformer, a cyst phantom is simulated. The radius of the cyst is $4 \mathrm{~mm}$ and is positioned at $35 \mathrm{~mm}$ on the axial axis. Random speckle pattern with the Gaussian amplitude is considered in default of software samples. The image is illustrated with $60 \mathrm{~dB}$ dynamic range. The results are shown in Figure 3. In the out of cyst area, the information is kept by
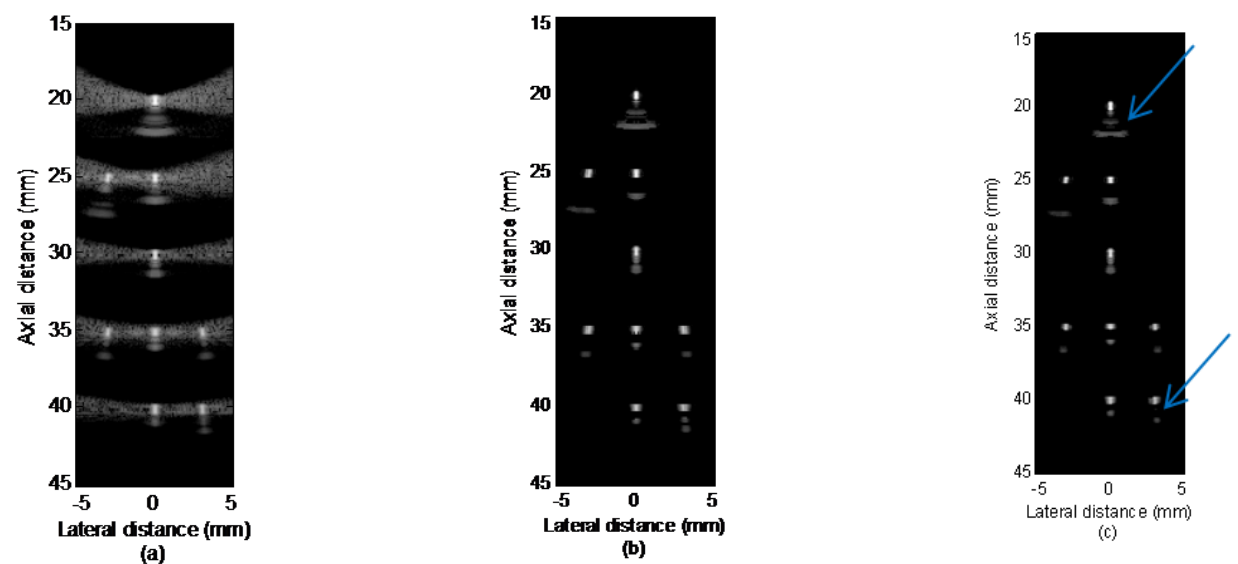

Fig. 2. Point target simulation. (a) the MV, (b) the ESBMV $(\partial=0.5)$, (c) the proposed method $(\Delta=0.1, m=1)$. 
proposed method. Some of areas are pointed by cycles in Figure 3.

For the ESBMV beamformer, the MV based beamformer and the proposed beamformer, contrast ratio (CR) and contrast-to-noise ratio (CNR) are estimated in the cyst phantom simulation. The CR is the ratio of the mean value in the background to the mean value in the cyst phantom [14], and the CNR is defined as the CR divided by the standard deviation of image intensity in the background region [15].

Results in Table 1 shows that the proposed method improves the contrast of the image.

\subsection{Phantom study}

Real data for the point target and cyst phantom imaging have been used. The cyst phantom imaging is also used to compare MV and ESBMV methods with the proposed method in term of the contrast enhancement. Central and sample frequencies are set to $5 \mathrm{MHz}$ and $40 \mathrm{MHz}$, respectively.

\subsubsection{Point target imaging}

To compare the resolution of the ESBMV method with the proposed method, six points are placed as shown in Figure 4 which illustrates the resulted image of the point target imaging experiment. The results were completely matched. It means that the proposed method kept the quality of the imaging resolution similar to the ESBMV beamformer.

\subsubsection{Cyst phantom imaging}

The data of a practical anechoic cyst was used for simulations as well. The cyst has a diameter of $6.7 \mathrm{~mm}$ and is centered at $(x, y, z)=(1.1,0,17.5) \mathrm{mm}$. The beamformer images are shown in Figure 5 with the dynamic range of $60 \mathrm{~dB}$.
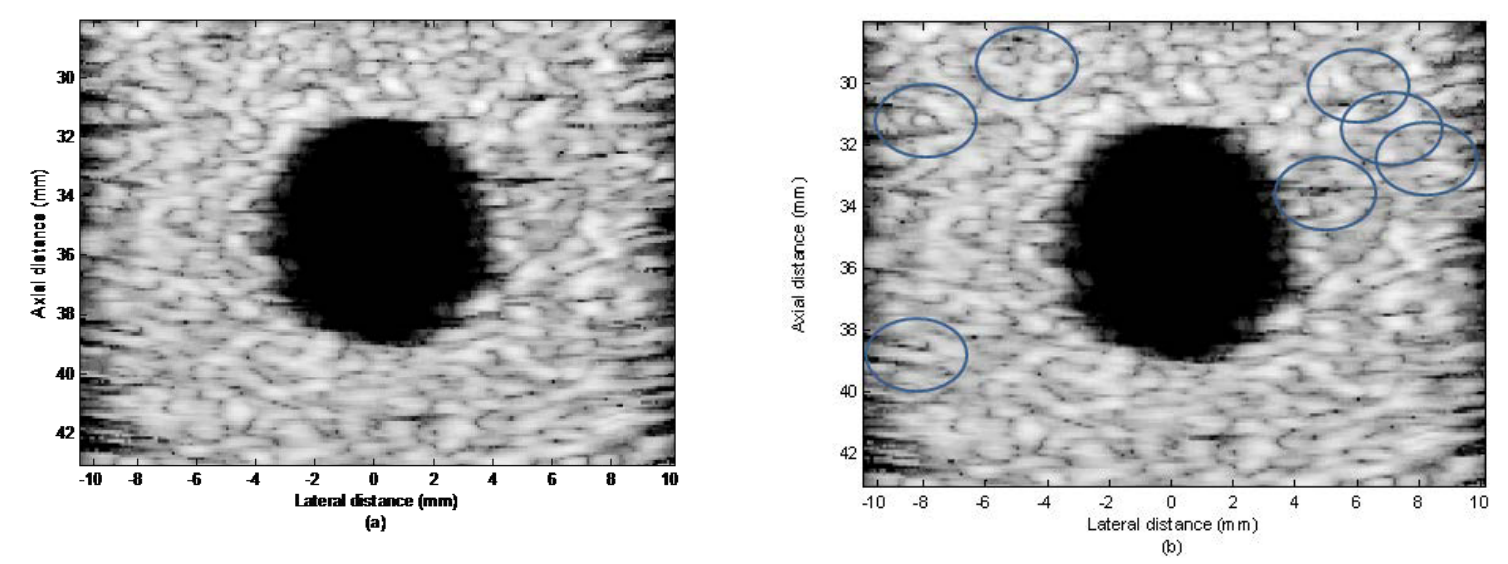

Fig. 3. The beamformed response for the simulated cyst phantom by using a) the $\operatorname{ESBMV}(\partial=0.5)$ b) the proposed $\operatorname{method}(\Delta=0.1, m=1)$.

Table 1

CR and CNR results of the MV, the ESBMV and the proposed beamformer for the cyst simulation

\begin{tabular}{lll}
\hline Beamformer & CR & CNR \\
\hline MV & 21.92 & 2.17 \\
ESBMV & 37.05 & 2.65 \\
Proposed Method & 37.4 & 2.76 \\
\hline
\end{tabular}



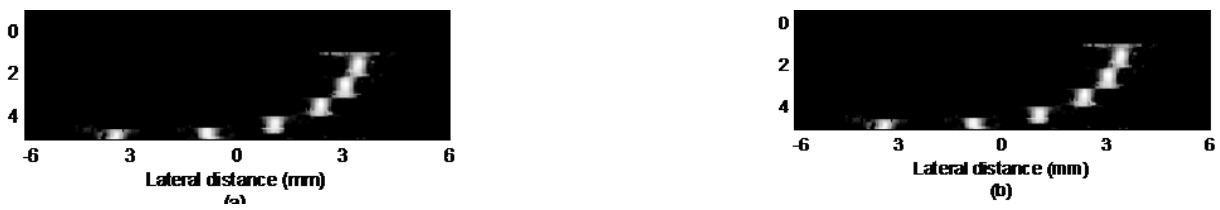

Fig. 4. Experiment results for the point target imaging by a) the ESBMV method and b) the proposed method.
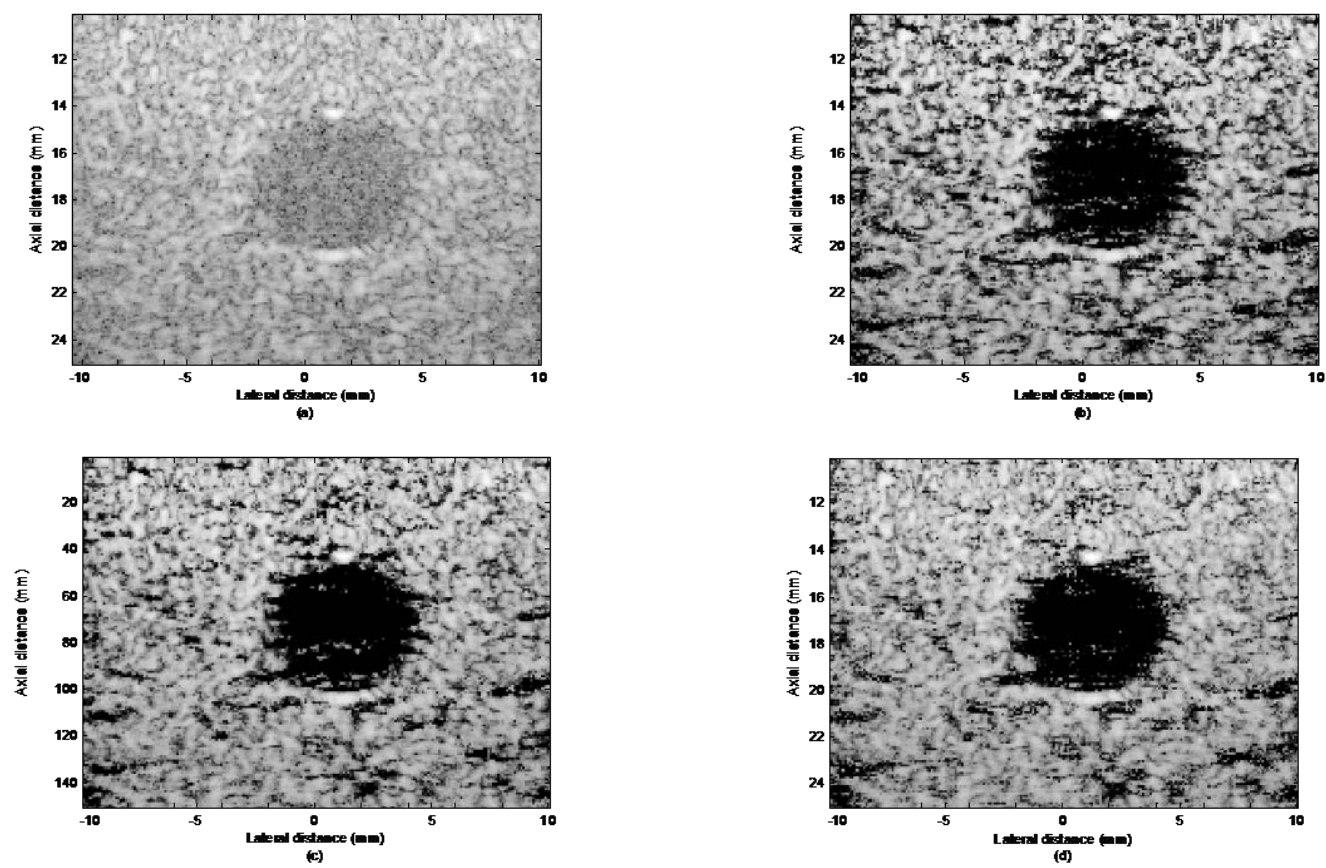

Fig. 5. The beamformed response for the real cyst phantom by using a) the MV, b) the ESBMV $(\partial=0.28)$, c) the proposed method $(\Delta=0.1, m=1)$ and d) the proposed method $(\Delta=0.1, m=3)$.

Table 2

$\mathrm{CR}$ and CNR results of the MV, the ESBMV and the proposed beamformer

\begin{tabular}{lll}
\hline Beamformer & CR & CNR \\
\hline MV & 8.88 & 1.01 \\
$\operatorname{ESBMV}(\partial=0.28)$ & 31.18 & 1.68 \\
Proposed Method $(m=1)$ & 32.66 & 1.78 \\
Proposed Method $(m=3)$ & 34.5 & 2.16 \\
\hline
\end{tabular}

For the ESBMV beamformer $\partial=0.28$ and for the proposed method $\Delta=0.1$ and $m=1$ and $\mathrm{m}=3$ were considered. Figure 5 show the real cyst phantom imaging results for the MV, the ESBMV and the proposed method. As it is shown, the proposed beamformer suppresses the interface component of points that are placed into the cyst area and keeps the signal information of points that are placed out of the cyst.

It has been shown that the performance of the proposed beamformer for real data is much better than that for simulated data. Referring to the results in Table 2, CR and CNR values are improved significantly. 


\section{Conclusion}

The proposed method provides the enhancement in the imaging contrast of PCA based beamformers significantly. By using echo signals of neighbor points, the interface component of echo signals was suppressed and the signal component was saved. Real target point simulation results show that the proposed beamformer is able to keep the quality of the imaging resolution, similar to the ESBMV beamformer. Simulated and real cyst phantom imaging results have also reflected the high performance of the proposed beamformer in terms of the imaging contrast improvement.

\section{Acknowledgment}

This work is supported by the National Basic Research Program of China (2015CB755500) and the National Natural Science Foundation of China (61271071, 61401102 and 61471125).

\section{References}

[1] J. Capon, High resolution frequency-wavenumber spectrum analysis, Proceedings of IEEE 57 (1969), 1408-1418.

[2] J.-F. Synnevåg, A. Austeng and S. Holm, Benefits of minimum variance beamforming in medical ultrasound imaging, IEEE Transaction on Ultrasonic, Ferroelectric and Frequency Control 56 (2009), 1868-1879.

[3] M. Sasso and C. Cohen-Barcie, Medical ultrasound imaging using the fully adaptive beamformer, Proceedings of IEEE International Conference on Acoustics, Speech, and Signal Processing 2 (2005), 489-492.

[4] J.-F. Synnevåg, A. Austeng and S. Holm, Adaptive beamforming applied to medical ultrasound imaging, IEEE Transaction on Ultrasonic, Ferroelectric and Frequency Control 54 (2007), 1606-1613.

[5] B.M. Asl and A. Mahloojifar, Eigenspace-based minimum variance beamforming applied to medical ultrasound imaging, IEEE Transactions on Ultrasonic, Ferroelectric Frequency Control 57 (2010), 2381-2390.

[6] B.M. Asl and A. Mahloojifar, Contrast enhancement and robustness improvement of adaptive ultrasound imaging using forward-backward minimum variance beamforming, IEEE Transactions on Ultrasonic, Ferroelectric Frequency Control $\mathbf{5 8}(2011), 857-867$.

[7] S. Mehdizadeh, T. Johansen and S. Holm, Eigenspace based minimum variance beamforming applied to ultrasound imaging of acoustically hard tissues, IEEE Transaction on Medical Imaging 31 (2012), 1912-1921.

[8] A. Rabinovich, A. feuer and Z. Friedman, Multi-line transmission combined with minimum variance beamforming in medical ultrasound imaging, IEEE Transactions on Ultrasonic, Ferroelectric Frequency Control 62 (2015), 814-827.

[9] X. Zeng, Y. Wang and J. Yu, Beam-domain Eigen space-based minimum variance beamformer for medical ultrasound imaging, IEEE Transaction on Ultrasonic, Ferroelectric and Frequency Control 60 (2013), 2670-2676.

[10] K. Kim, S. Park, J. Kim, S.B. Park and M. Bae, A fast minimum variance beamforming using principal component analysis, IEEE Transactions on Ultrasonic, Ferroelectrics, and Frequency Control 61 (2014), 930-945.

[11]B.M. Asl and A. Mahloojifar, A low-complexity adaptive beamformer for ultrasound imaging using structured covariance matrix, IEEE Transactions on Ultrasonic, Ferroelectric Frequency Control 59 (2012), 660-667.

[12] R.A. Monzingo and T.W. Miller, Introduction to Adaptive Arrays, Wiley, New York, 1980.

[13] J.A. Jensen, Field: A program for simulating ultrasound systems, Medical \& Biological Engineering \& Computing 34 (1996), 351-354.

[14]M. O'Donnell and S.W. Flax, Phase-aberration correction using signals from point reflectors and diffuse scattersmeasurements, IEEE Transactions on Ultrasonic, Ferroelectric Frequency Control 35 (1988), 768-774.

[15] S. Krishnan, K.W. Rigby and M. O'Donnell, Improved estimation of phase aberration profiles, IEEE Transactions on Ultrasonic, Ferroelectric Frequency Control 44 (1997), 701-713. 